\title{
EDGE DETECTION IN ATOMIC MAGNETOMETER IMAGING
}

\section{N. Papi, E. Cali, C. Marinelli, E. Mariotti, V. Millucci}

\section{Department of Physical Sciences, Earth and Environment, Siena University, Siena, Italy}

\begin{abstract}
We present the results of an edge detection algorithm applied on Electromagnetic Induction Imaging provided by an Atomic radio-frequency Magnetometer operating in an unshielded environment and at room temperature. Atomic Magnetometers have been already used for Imaging Techniques in the last few years, but the image reconstruction and the object pattern recognition lacks nowadays in terms of quality: the effect of scattering of e.m. signals at low-frequency provides blurred images, and does not allow for a clean ray - optics response, as in the case of X rays. Our algorithm, based on solved Gaussian Noise Recognition, demonstrates excellent spatial resolution achieved despite low Signal-to-Noise-Ratio.
\end{abstract}

Key words: Magnetometry; All-optical sensors; Statistical Data analysis; Gaussian Noise

\section{INTRODUCTION}

During the last few years it has been proved that Atomic Magnetometers represent a valid alternative to traditional Imaging Techniques. They are safer and healthier than $\mathrm{X}$ - ray systems, as do not use ionizing radiation; they down scale in magnetic fields for 9 orders of magnitudes, operating at nanoTesla instead of Tesla values, typical of Nuclear Magnetic Resonance (NMR), and allow for the construction of portable sensors/detectors. In comparison with the non-optical magnetometers, they have better sensibility and (at least in the radiofrequency range) better tunability. Finally, working around room temperature, they do not need high costs of maintenance, as in the case of the SQUIDs, where cryogenics is essential; moreover, they do not need costly and bulky mu - metal shielding.

Electromagnetic induction imaging is a non-invasive method that can investigate the conductivity or permittivity properties of a media. It is based on the detection of the secondary field yield by surface eddy currents, when induced by an external oscillating magnetic field. An experimental setup has been recently built at University College of London (UCL), where very interesting results have been obtained and reported. ${ }^{1,2}$

Nowadays several methods of edge detection have been developed, essentially based on changes in brightness or discontinuities in depth. All these techniques massively manipulate the original image data causing a loss of information; moreover, all the derivative-based methods do not guarantee good performances when very noisy signals are taken into account. Any additional smoothing procedure will change the original data.

We present in this paper a procedure where attention is put on background noise in such a way to avoid any contamination or alteration of the original data, as given by UCL group.

\section{EXPERIMENTAL SETUP}

The experimental setup for the detection of metallic samples via an optical method has been built at University College of London (UCL); a simplified scheme is shown in Figure 1. A Distributed Bragg Reflector (DBR) Laser provides the light beam, being locked in frequency by a control on a ${ }^{87} \mathrm{Rb}$ reference cell that guarantees the tuning of the laser with the $\mathrm{D}_{2}$ line of Rubidium in vapor phase.

The core of the apparatus is a second Rubidium atom cell, exposed to an external magnetic field and to the action of the laser light. The laser beam is divided by a Beam Splitter (BS); the more intense beam is circularly $(\sigma)$ polarized, exciting ${ }^{87} \mathrm{Rb}$ atoms in the sensor cell on a closed hyperfine transition by optical pumping. In fact, the population of the atomic Zeeman states is deeply modified, due to the specific polarization of the laser. The effect of this beam is therefore a net polarization of the atomic sample. The second beam, which is used as a probe beam (low intensity beam), is linearly $(\pi)$ polarized; Faraday Effect rotates its polarization in the passage through the polarized atomic vapor. In this way the light measures the status of polarization of the sample in a very sensitive

Correspondence to:

E. Mariotti

Department of Physical Sciences, Earth and Environment, Siena University, via Roma 56, 53100 Siena, Italy.

Tel.: +39.0577.234684

E-mail: mariotti@unisi.it 
detection scheme. The ${ }^{87} \mathrm{Rb}$ cell is put in a pretty uniform magnetic field $\mathrm{B}_{\mathrm{DC}}$ provided by two coils in Helmholtz's configuration.

Along the coils symmetry axis, a movable single ferrite coil driven by a radiofrequency (RF) current at $1 \mathrm{kHz}$ generates a $\mathrm{B}_{\mathrm{RF}}$ magnetic field. This time dependent field 1) produces a position - dependent redistribution of the population in the atomic levels and 2) it is able to induce eddy currents on the surface of a metallic sample, the object to be detected. These currents are responsible for a supplementary magnetic field, which changes the optical signal associated to the probe beam. A dual-phase Lock-In Amplifier (LIA), referenced on the RF of the ferrite coil, collects the amplitude of the magnetic field signal (R-map) as detected by the probe optical beam and its phase-lag

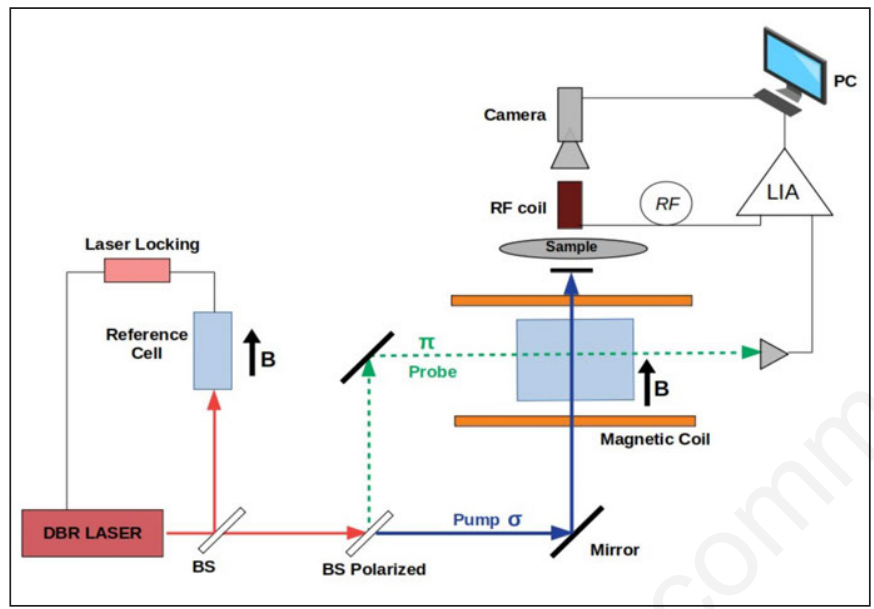

Figure 1. Simplified experimental setup.

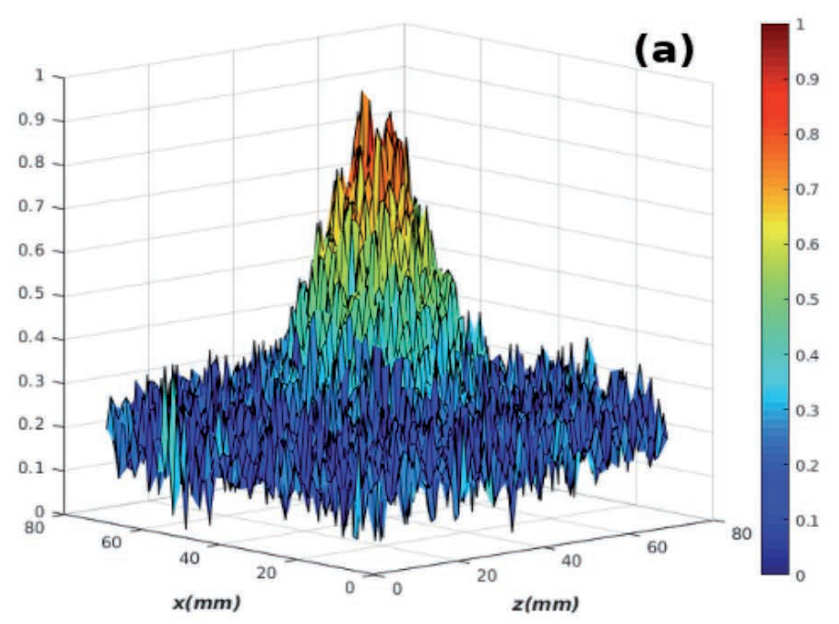

(-map) depending on the position of the ferrite coil providing us a collection of data that can be rendered in a 2D map of the total signal.

\section{GAUSSIAN NOISE HYPOTHESIS AND CHARACTERIZATION}

A typical normalized data acquisition run is reported in Figure 2 at two different angles of view: the presence of a $30 \mathrm{~mm}$ diameter copper disk is clearly detected, but the edge of the object cannot be distinctly assigned. The major factor of this limited definition is given by the diameter of the RF coil (we would like to have an ideal point source); in this experiment the coil is $8 \mathrm{~mm}$ and this value determines the spatial resolution of the setup. In order to clean up the image from noise, it is useful to perform a histogram image transformation. By this method, it is reported how many sectors of the explored area $(70 \times 70) \mathrm{mm}^{2}$ fall in a certain interval of the signal values, defined as bin after normalization. For the sake of simplicity, we have considered 100 different bins, corresponding to a separation in intensity value of 0.01 for each bin.

The histogram image of the previous picture is reported in Figure 3. The graphics shows a nonsymmetric behavior with a calculated skewness of 1.87 . It can be obtained as the sum of two contributions: the first one is a Gaussian-like curve centered around 0.25, describing the noise values, and a long tail towards the highest values, where the disk is present. This second contribution is the reason for the asymmetry. After these considerations, it becomes evident that the borders of the sample are located at the right side of the Gaussian curve, exactly where the symmetry is broken from secondary fields on the surface of the sample.

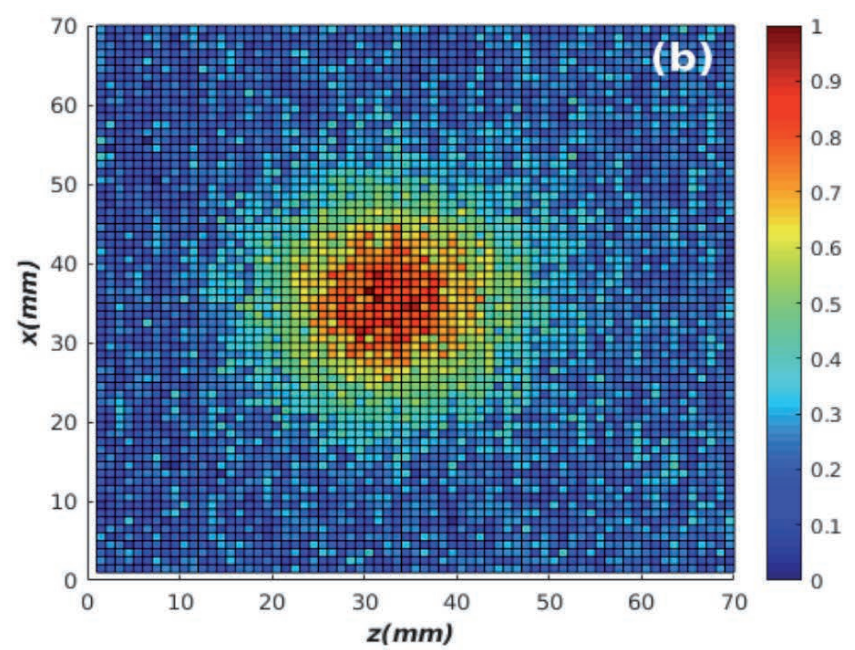

Figure 2. Normalized conductivity R-map of a $30 \mathrm{~mm}$ diameter Copper Disk represented in 3D (a) and 2D (b). The edges of the sample do not clearly emerge from the background noise. 
Although a Gaussian Noise hypothesis is the most generic behavior for a background signal, it is necessary to perform a hypothesis test before proceeding with the analysis, because the cleaning procedure will be strongly depend from the kind of the statistical curve introduced for the Noise Characterization.

Considering now a single row or column at the border of the $(70 \times 70) \mathrm{mm}^{2}$ matrix of collected data, where any contribution due to secondary field from the sample is minimized, we have to check if the distribution of the values follows a Gaussian distribution. Defining the mean and the standard deviation of the selected data as usual:

$$
\mu=\sum_{i=1}^{N} \frac{x_{i}}{N} \sigma=\sum_{i=1}^{N} \sqrt{\frac{\left(\mu-x_{i}\right)^{2}}{N-1}}
$$

We can reconstruct the Normal Distribution with the same mean and standard deviation of the noise dataset. In Figure 4 the Cumulative Distribution for the noise data and for the theoretical gaussian hypothesis is shown. The two curves are overlapped, showing the same behavior: however, a Kolmogorov - Smirnov statistical test has been performed, as a quantitative test as previously reported. ${ }^{3}$ The obtained $\mathrm{P}$ value is 0.857 , rejecting the alternative hypothesis that the two distributions have different behaviors.

Another graphical method to test normal distributions, ${ }^{3}$ is called Quantile-Quantile Plot (Q-Q Plot) and it consists in plotting the quantiles of experimental cumulative distributions versus Normal Curve Quantiles. In Figure 3, on the right, the experimental points are reported in blue together with the Linearized Normal Distribution, in red, as a graphical confirmation of the Kolmogorov - Smirnov result.

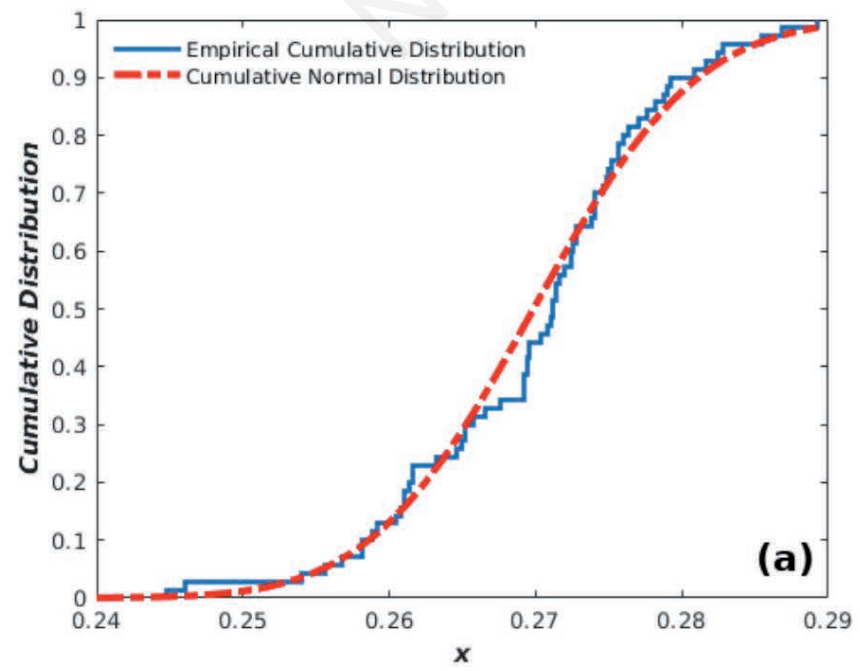

All the statistical tests performed in different samples of data and different conductivity maps do not reject the Gaussian-Noise hypothesis.

After the confirmation of the Gaussian Behavior of the noise, a gaussian fitting method, based on nonlinear least square treatment, has been employed on histogram data, in order to find the best parameters that describe the noise behavior.

As the total histogram data does not have a Normal behavior due to the presence of the disk, the fitting procedure cannot be applied on the entire curve but

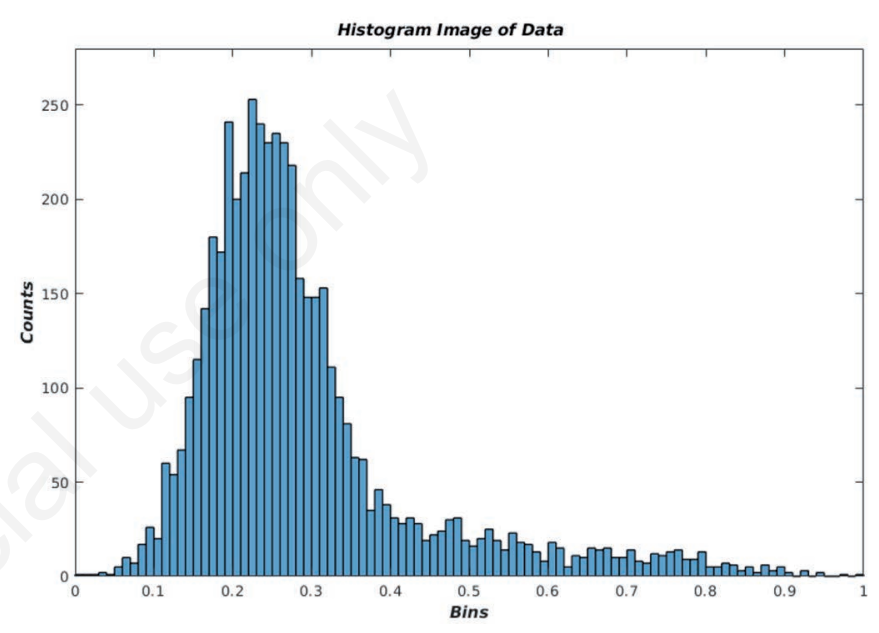

Figure 3. Histogram Image of R-map of the $30 \mathrm{~mm}$ diameter Copper Disk. The curve centered about 0.25 shows an almost Gaussian behavior, the asymmetry in higher bins values depends on the presence of the conductive sample.

Figure 4. (a) The Cumulative Noise Distribution (blue) vs the Cumulative Normal Distribution with same mean and standard deviation (red) for the R-map of Cu Disk. (b) QQ Plot for noise data from the R-map of 30 mm diameter Copper Disk. The experimental points show a linear behavior, confirming the Gaussian Distribution for the noise. 
only in first part where the curve presents a Gaussian behavior, as confirmed by hypothesis tests.

Selecting a restricted range where only a single fit curve is introduced is not recommended because it inserts a threshold to be arbitrarily specified. This value could be partially undetermined or even totally wrong. In order to avoid this problem, an iterative fitting process has been implemented in the algorithm; it considers several histograms ranges where the fits can be calculated. In order to select the best parameters, resulting from the best fit performed, it is necessary to introduce a merit figure as the root-mean-square-error (RMES), defined as:

$$
R M S E=\sqrt{\sum_{i}^{N} \frac{\left(x_{o_{i}}-x_{p_{i}}\right)^{2}}{\gamma}}
$$

where $\mathrm{x}_{\mathrm{o}}$ and $\mathrm{x}_{\mathrm{p}}$ are the observed and the predicted counts for each bin interval and represents the number of degrees of freedom of the fit.

It is evident that the best fit procedure yields the lower RMSE and in this way we can extrapolate the best gaussian parameters describing the noise behavior. A typical fit is shown in Figure 5.

\section{RESULTS}

Knowing the Gaussian Noise parameters, i.e. the amplitude $A$, the mean $\mu$ and the standard deviation $\sigma_{\mathrm{G}^{\prime}}$ as obtained from the fit method, it becomes possible to remove noise contributions in the original data implementing a threshold in terms of Gaussian Noise's standard deviation, in this case $T=3 \sigma_{\mathrm{G}}$. Higher threshold values correspond to both a less noisy final image and a reduction of the size of the sample; on the contrary, at lower threshold value the final image results more noisy. Even though there is an unknown relationship between the signal-to-noise-ratio (SNR) and the threshold value to be chosen, good results are obtained with an empirical ratio of $T=3 \sigma_{G^{\prime}}$ when $\mathrm{SNR}=4$; in fact, this value statistically removes $99 \%$ of the noise contribution, at least in our case.

The cleaned image is shown in Figure 6. As com-

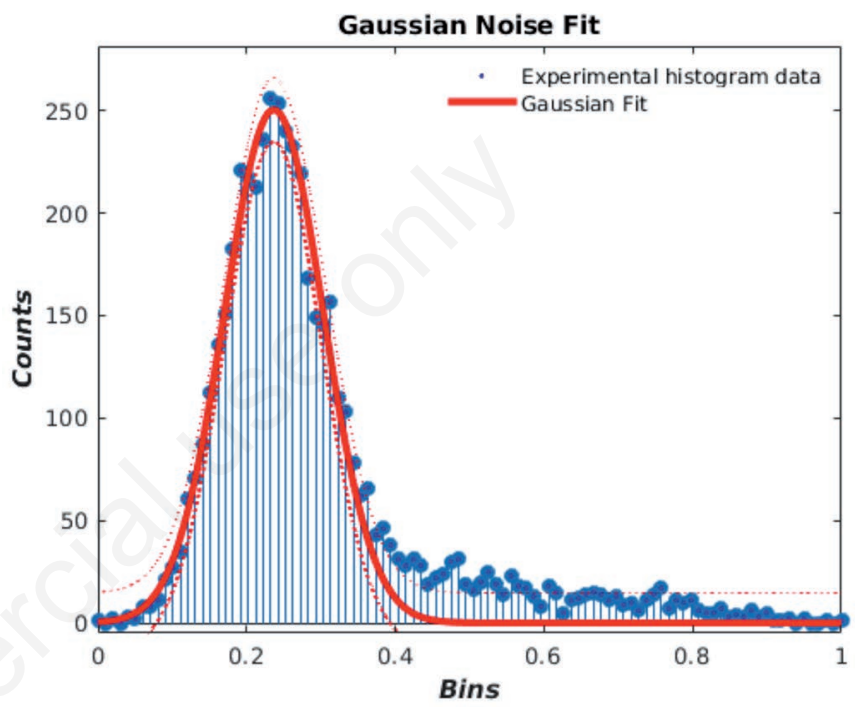

Figure 5. Gaussian fit on the noise contribution on the histogram image for R-map of the $30 \mathrm{~mm}$ diameter Copper Disk. The fit, minimizing the RMSE, give us the best parameters to characterize the noise.
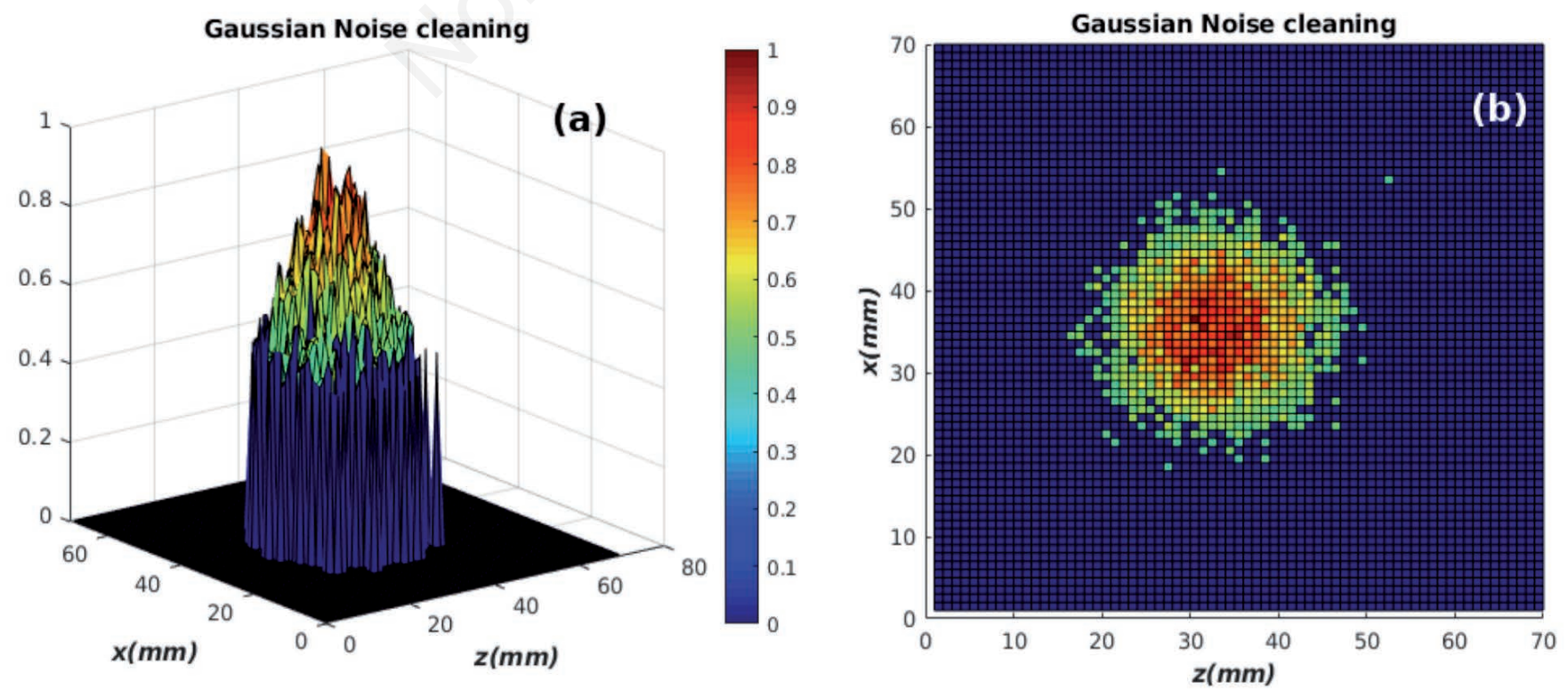

Figure 6. R-map of the $30 \mathrm{~mm}$ diameter Copper Disk after the noise removal. (a) 3D reconstruction. (b) surface plot. The edges of the disk are clearly visible, as compared to the original data. 
pared to original data of Figure 2, the cleaning procedure massively removes the noise background emphasizing the edge of the sample. Moreover, as we already stressed in the previous paragraphs, our algorithm does not have any impact onto original data avoiding possible losses of original information.

The residual noise contribution is randomly dispersed around the sample, as it is evident in Figure 6, and can be cleaned with more traditional method as the "convolution masking".

The convolution masking technique changes each value of the raw matrix with the mean of its neighbors smoothing the noise value. Because the outliers are randomly dispersed and they are isolated from each other, the mean around their position is dramatically lower with respect to the edges of the sample, where convolution masking technique is essentially useless. The masking method anyway modifies the original data causing a loss of original information, and it has to be used with caution or in the final step of algorithm as in this case. However, if the SNR is sufficiently high, bigger threshold can be selected in order to avoid the implementing of masking method.

The final result of this algorithm for the R-map of a $30 \mathrm{~mm}$ diameter Copper Disk is shown in Figure 7, where a boundary blue line surrounds the sample's points selected from algorithm. An additional circle fit has been added in order to estimate the coordinates of the center of the copper disk. The effective radius results $r_{e}=16 \pm 1 \mathrm{~mm}$, in agreement with the real size (15 $\mathrm{mm})$. In Figure $7 \mathrm{~b}$, it is possible to compare the result of the algorithm circle fit, when overlapped with the raw data. The edges of the disk place at values around
0.4, corresponding to the tail of Noise Gaussian Characterization previously described and shown in Figures 3 and 5.

Similar results have been obtained on the Phase map, as shown in Figure 8. Anyway, this kind of signal shows a considerably lower SNR, due to the field gradient induced by the non - point like coil. In fact, the selected disk points, as defined by the statistical procedure, reveal the same strong asymmetry as shown by the raw data. Even though the fitted curve circle does not trace the boundary of the real sample, the effective radius $r_{e}=16 \pm 1 \mathrm{~mm}$ has been extracted, as in $\mathrm{R}$-map signal, and the sample is clearly detected.

In order to test the sensitivity of the algorithm and of the overall setup, a sub-millimeter crack has been created in an Aluminum ring. In the region of the crack the secondary field around it should be absent because the eddy currents are suppressed by the dielectric interspace. In Figure 9b the data from the Optical Magnetometer are shown: it is clearly evident the drop of the signal field values in correspondence of the cut in the ring. The bigger dimension of the coil with respect to the crack does not permit a full resolution of the defect, but the MRI and the analysis algorithm are able to detect details under $1 \mathrm{~mm}$ in size.

\section{CONCLUSIONS}

The analysis method applied to Magnetometer Resonance Imaging allows for the detection of a conductor sample in the space after a characterization of the noise background both from the R-map signal
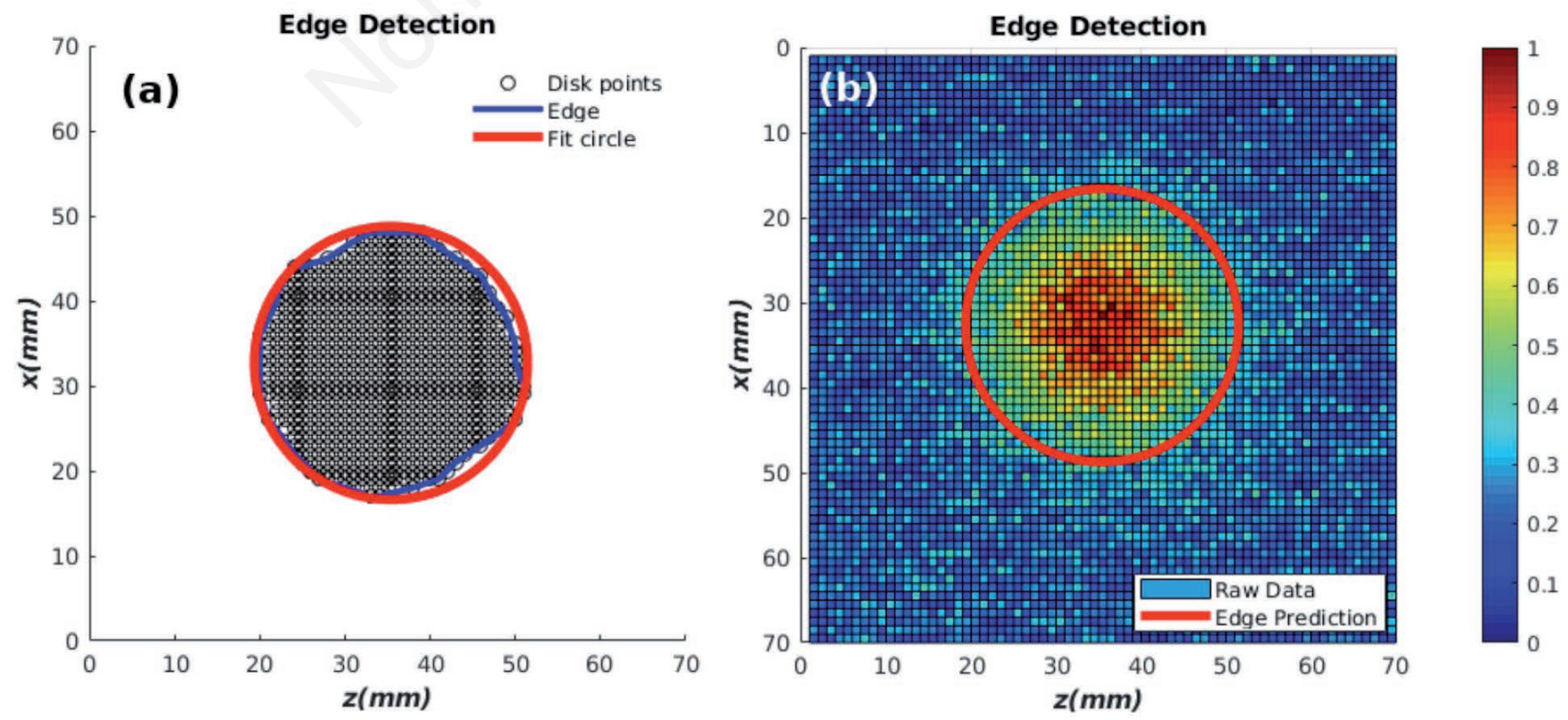

Figure 7. Edge detection of the R-map of the 30mm diameter Copper Disk: (a) the algorithm selected area delimited by a blue line (the relative best circle fit is in red); (b) the overlap of collected original data with the calculated fit in red. 
and the P-map one. We demonstrated its performance in determining the size of the sample and in tracing borders' sample with an error lower than the dimension of the RF coil, used as a non - point like source for the atomic sensor. Moreover, it should be noted that the experimental setup and the analysis method achieved a sensitivity smaller than or equal to $1 \mathrm{~mm}$ in size in an unshielded environment.

The analysis procedure does not have impact on the original data and it avoids any loss of information in order to apply other or more sophisticated imaging techniques.
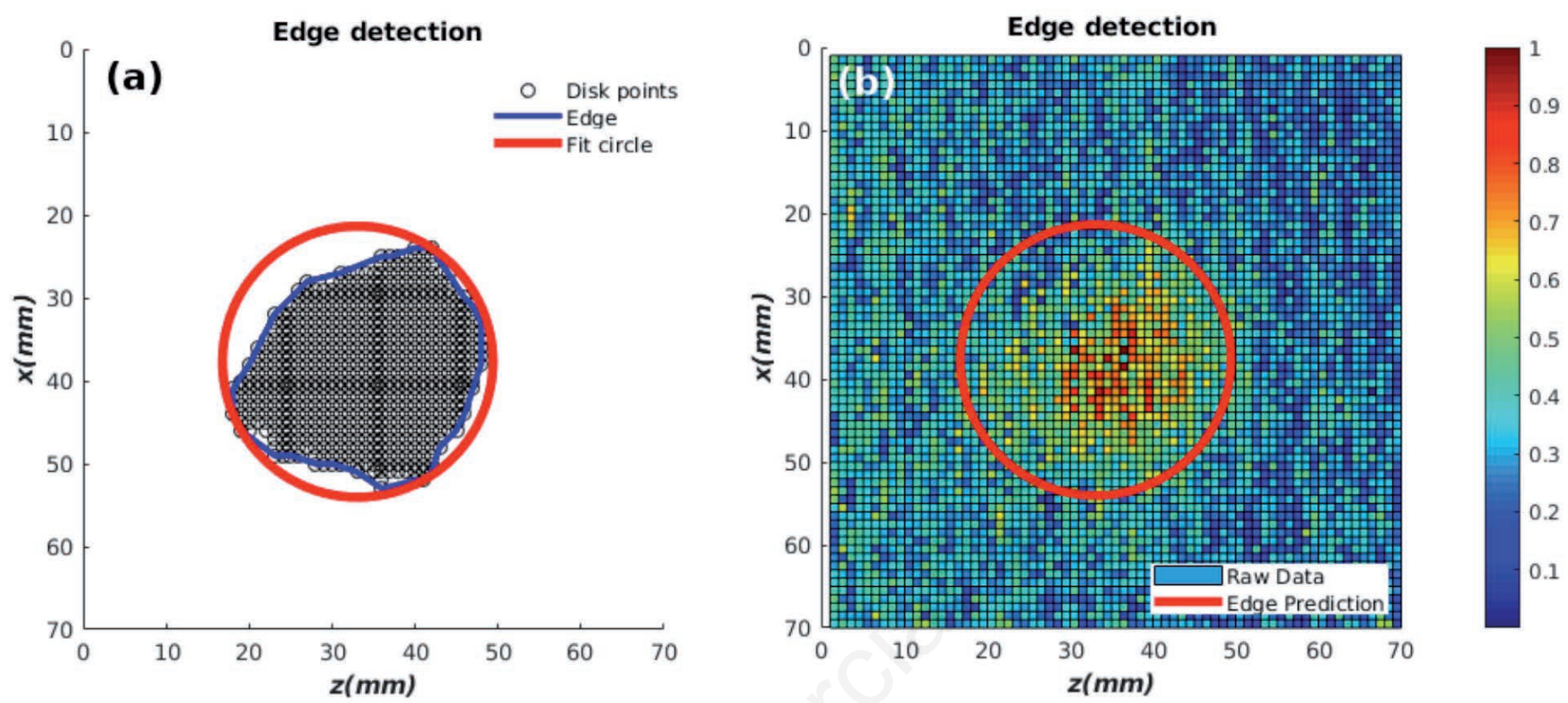

Figure 8. Edge detection of P-map of the $30 \mathrm{~mm}$ diameter Copper Disk: (a) the algorithm selected area delimited by a blue line (the relative best circle fit is in red); (b) the overlap of collected original data with the calculated fit in red.
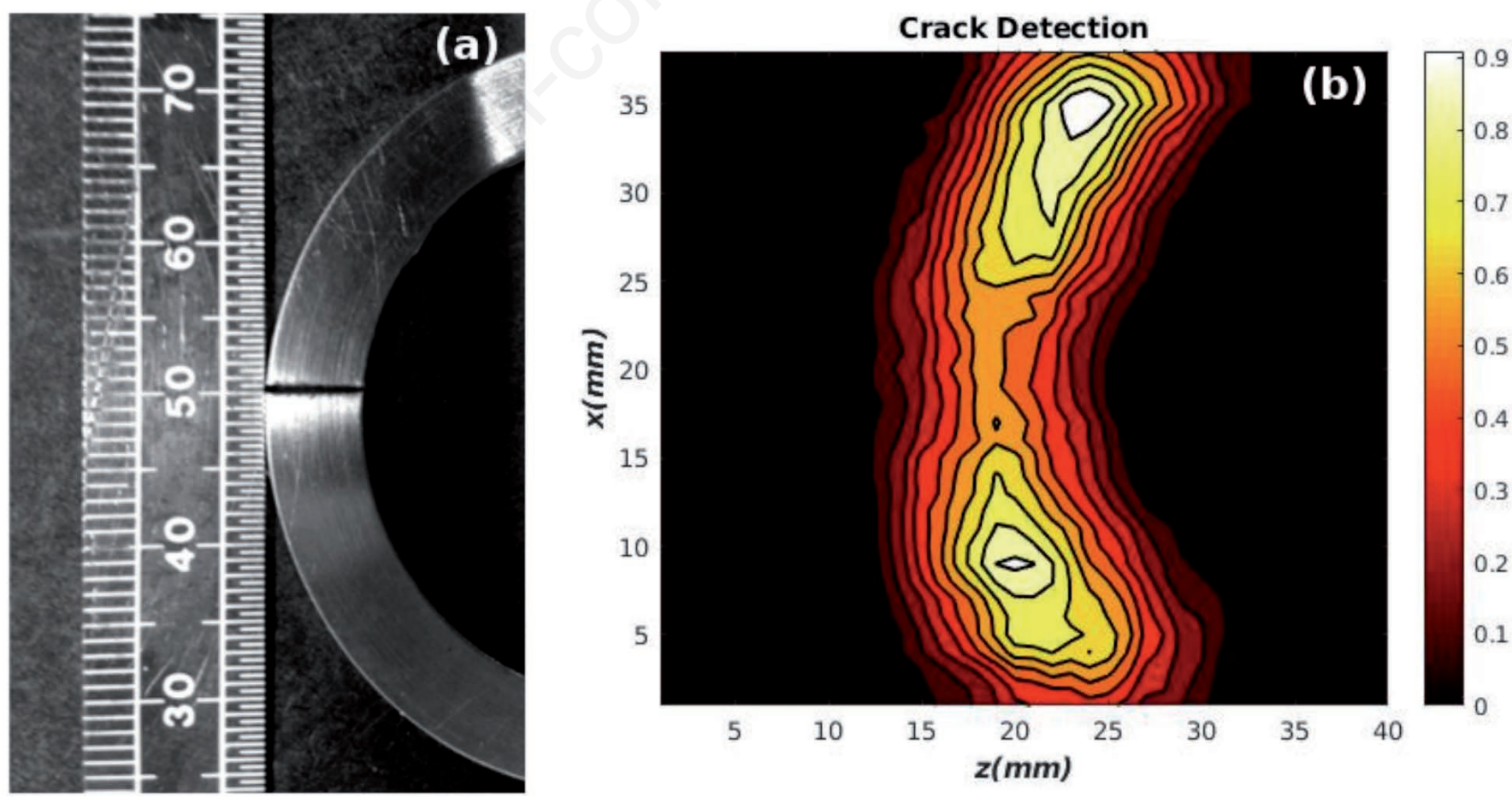

Figure 9. (a) Picture of the induced sub-millimeter crack on Aluminum Ring. (b) The R-map conductivity, where it is evident an important decrease in the secondary field around the crack zone. 


\section{ACKNOWLEDGEMENTS}

The authors wish to thank F. Renzoni and L. Marmugi for the MRI data and L.Marmugi and T. Del Prete for very fruitful discussions.

\section{REFERENCES}

1. Deans C, Marmugi L, S. Hussain, Renzoni F. Electromagnetic induction imaging with a radio-frequency atomic magnetometer. Appl Phys Lett 2016;108:103503.

2. Deans C, Marmugi L, S. Hussain, Renzoni F. Optical atomic magnetometry for magnetic induction tomography of the heart. Proc SPIE 9900. 2016. Quantum Optics, 99000F.

3. Del Prete T. Methods of Statistical Data Analysis in Particle Physics. Istituto Nazionale di Fisica Nucleare, Sezione di Pisa, Italy. 28 May 2000. Available from: www.pg.infn.it/l3/biasini/dott/delprete.ps 